\title{
Haptoglobin Genotype-Dependent Anti-Inflammatory Signaling in $\mathrm{CD}_{163}{ }^{+}$Macrophages
}

\author{
R. Clive Landis, ${ }^{1,2}$ Pandelis Philippidis, ${ }^{2}$ Jan Domin, ${ }^{3}$ \\ Joseph J. Boyle, ${ }^{2}$ and Dorian O. Haskard ${ }^{2}$ \\ ${ }^{1}$ Edmund Cohen Laboratory for Vascular Research, Chronic Disease Research Centre, \\ The University of the West Indies Bridgetown BB11115, Barbados \\ ${ }^{2}$ Eric Bywaters Centre for Vascular Inflammation, Faculty of Medicine, Imperial College London, London W12 0NN, UK \\ ${ }^{3}$ Department of Life Sciences, University of Bedfordshire, Luton LU1 3JU, UK
}

Correspondence should be addressed to R. Clive Landis; clive.landis@cavehill.uwi.edu

Received 15 March 2013; Accepted 3 April 2013

Academic Editor: David A. Hart

Copyright ( 2013 R. Clive Landis et al. This is an open access article distributed under the Creative Commons Attribution License, which permits unrestricted use, distribution, and reproduction in any medium, provided the original work is properly cited.

\begin{abstract}
Intraplaque hemorrhage causes adaptive remodelling of macrophages towards a protective phenotype specialized towards handling iron and lipid overload, denoted Mhem. The Mhem phenotype expresses elevated levels of hemoglobin (Hb) scavenger receptor, $\mathrm{CD} 163$, capable of endocytosing pro-oxidant free $\mathrm{Hb}$ complexed to acute phase protein haptoglobin (Hp). It is notable that individuals homozygous for the Hp 2 allele (a poorer antioxidant) are at increased risk of cardiovascular disease compared to the Hp 1 allele. In this study, we examined whether scavenging of polymorphic Hp:Hb complexes differentially generated downstream anti-inflammatory signals in cultured human macrophages culminating in interleukin (IL)-10 secretion. We describe an anti-inflammatory signalling pathway involving phosphatidylinositol-3-kinase activation upstream of Akt phosphorylation (pSer473Akt) and IL-10 secretion. The pathway is mediated specifically through CD163 and is blocked by anti-CD163 antibody or phagocytosis inhibitor. However, levels of pSer473Akt and IL-10 were significantly diminished when scavenging polymorphic $\mathrm{Hp} 2-2: \mathrm{Hb}$ complexes compared to Hp1-1:Hb complexes $(P<0.05)$. Impaired anti-inflammatory macrophage signaling through a CD163/pAkt/IL-10 axis may thus represent a possible Hp2-2 disease mechanism in atherosclerosis.
\end{abstract}

\section{Introduction}

Intraplaque hemorrhage is a common complication of atherosclerosis and is linked to plaque progression, especially in diabetes [1-4]. However, work from independent groups has demonstrated that macrophages at zones of hemorrhage may exert some level of homeostatic control through adaptive remodelling towards an Mhem phenotype capable of handling iron and lipid overload [5-7]. Scavenging of haptoglobin:hemoglobin ( $\mathrm{Hp}: \mathrm{Hb})$ complexes via $\mathrm{CD} 163$ is part of this adaptive process, linked to secretion of antiinflammatory cytokine interleukin (IL)-10 and elevation of heme oxygenase (HO)-1 [8-10]. Analogous protective pathways are evoked by free diffusion of purified heme or by phagocytosis of damaged erythrocytes via CD204, leading to the proposal that a final common pathway is instigated by accumulation of intracellular heme capable of transcriptionally activating genes involved in iron handling and cholesterol efflux (e.g., HO-1 and liver X receptor) via transcription factors Nrf2 and activating transcription factor (ATF)-1 [11, 12]. Although the atheroprotective properties of the Mhem macrophage phenotype is therefore well established, the role of proximal signalling pathways linked to antiinflammatory IL-10 secretion via CD163-dependent uptake of $\mathrm{Hb}: \mathrm{Hp}$ remains to be fully understood.

In addition to IL-10, immunoregulatory IL-6 cytokine has been consistently reported downstream of CD163 $[8,13,14]$. However, the earliest IL- 6 studies using cross-linking antiCD163 antibodies may not have adequately discriminated between surface versus endocytosis-dependent effector pathways $[13,14]$. Whether downstream signals require simple cross-linking of CD163 at the cell surface or phagocytosis 
of the entire $\mathrm{Hp}: \mathrm{Hb}$ complex is an important distinction, since Hp2-2:Hb binds more avidly than Hp1-1:Hb to CD163 at the surface but, conversely, is more poorly internalised into the cell $[15,16]$. More recent investigations employing native $\mathrm{Hp}: \mathrm{Hb}$ ligand appeared to suggest poor or even lack of dependence on CD163 for IL-6 or IL-10 signalling pathways, depending on the type of polymorphic haptoglobin variant employed [17, 18]. Since the haptoglobin 2 allele is linked to a host of adverse clinical cardiovascular events, [19-23] it is important to understand Hp genotype-dependent disease mechanisms in $\mathrm{CD}_{163}{ }^{+}$macrophages in greater detail, to guide informed interdictions in vulnerable individuals.

Here we have examined IL-10 signalling pathways during scavenging of polymorphic $\mathrm{Hp} 2-2: \mathrm{Hb}$ versus $\mathrm{Hp1-1:Hb}$ complexes in $\mathrm{CD}_{163}{ }^{+}$human monocyte-derived macrophages. We identify a specific Akt/IL-10 pathway that is comparatively underinduced during the scavenging of $\mathrm{Hp} 2$ complexes.

\section{Materials and Methods}

2.1. Reagents and Antibodies. Human $\mathrm{Hb}\left(\mathrm{A}_{\mathrm{o}}\right)$, human $\mathrm{Hp}$ (phenotypes 1-1 and 2-2), and colchicine were purchased from Sigma-Aldrich (Poole, UK). Anti-human CD163 monoclonal antibody clones RM3/1, Ki-m8, and 5C6-FAT were purchased from Bachem (Merseyside, UK), clone GHI/61 from BD Pharmingen (Oxford, UK), and clone Ber-MAC3 from Dako (Cambridge, UK). Polyclonal anti-Akt and anti-phosphoAkt (Ser473) antibodies were purchased from Cell Signalling Technology, Inc. (Beverley, MA). The phosphoinositide-3kinase (PI-3K) inhibitor, Ly294002, was purchased from Alexis Corporation (Bingham, UK). Endotoxin determinations were made using the GCL-1000 LAL chromogenic endpoint assay (Cambrex Bio Science, Wokingham, UK).

2.2. Hb:Hp Treatment of Macrophage Cultures. Human monocytes were isolated from venous blood and differentiated into $\mathrm{CD} 163^{+}$macrophages in vitro as described [8]. Hb:Hp complexes were generated by dissolving equimolar amounts of $\mathrm{Hb}$ and $\mathrm{Hp}$ in growth medium. $\mathrm{Hb}, \mathrm{Hp}$, or $\mathrm{Hb}: \mathrm{Hp}$ were added at final concentrations of $1 \mathrm{mg} / \mathrm{mL}$ unless otherwise stated to monocyte/macrophage cultures prior to incubation for 24 hours and collection of supernatants and/or cell lysates for IL-10 and Akt analysis, respectively. $\mathrm{Hb}$ or $\mathrm{Hp}$ batches containing detectable endotoxin $(>5 \mathrm{pg} / \mathrm{mL})$ were discarded. Supernatants and cell lysates were stored in aliquots at $-70^{\circ} \mathrm{C}$ prior to analysis. In some experiments, the PI-3K inhibitor Ly294002 was added at 50, 25, 12.6, and $6.25 \mu \mathrm{mol} / \mathrm{L}$ final concentrations. Actinomycin D and cycloheximide were added at a concentration of $1 \mu \mathrm{g} / \mathrm{mL}$ and colchicine at a concentration range between 10 and $1.25 \mu \mathrm{mol} / \mathrm{L}$. None of the inhibitors at the concentrations used exhibited significant macrophage cellular cytotoxicity [24]. Anti-CD163 monoclonal antibodies were added at a final concentration of $20 \mu \mathrm{g} / \mathrm{mL}$, previously shown to be neutralizing [8].

2.3. Enzyme Linked Immunosorbent Assays. IL-10 concentrations in culture supernatants were determined by ELISA technique (Quantikine; R\&D Systems, Abingdon, UK) according to the manufacturer's recommendations. PhosphoAkt levels in cell lysates, collected in buffer consisting of $1 \%$ Triton X-100, $25 \mathrm{mmol} / \mathrm{L}$ sodium deoxycholate, $150 \mathrm{mmol} / \mathrm{L}$ $\mathrm{NaCl}, 50 \mathrm{mmol} / \mathrm{L}$ Tris $\mathrm{pH}$ 7.4, $4 \mathrm{mmol} / \mathrm{L}$ EDTA, $200 \mu \mathrm{mol} / \mathrm{L}$ sodium orthovanadate, $10 \mathrm{mmol} / \mathrm{L}$ sodium pyrophosphate, $100 \mathrm{mmol} / \mathrm{L}$ sodium fluoride, $1 \mathrm{mmol} / \mathrm{L}$ phenylmethylsulfonyl fluoride, and 5\% protease inhibitor cocktail (Sigma Aldrich), were measured by pSer473 Akt kit (Biosource, Camarillo, CA) according to manufacturer's instructions. All samples were measured in duplicate and results expressed as mean cytokine concentration $(\mathrm{pg} / \mathrm{mL}$ ) or pAkt activity $(\mathrm{U} / \mathrm{mg}) \pm \operatorname{SEM}$ from $n=3$ experiments.

2.4. Western Blot Analysis. Monocytes/macrophages were lysed in buffer, as described for the phospho-Akt ELISA, and proteins were separated by SDS-PAGE on a $12.5 \%$ gel prior to transfer to Immobilon-P membranes (Millipore Corporation, Bedford, MA). Equal loading of lanes was confirmed by estimation of lysate protein content using the Bio-Rad $D_{c}$ protein assay (Bio-Rad, Hercules, CA). Induction of pSer473Akt was established relative to total Akt by probing blots with polyclonal antibody against pSer473Akt, followed by stripping and reprobing with total Akt. Blots were developed with an enhanced chemiluminescence substrate (Amersham Pharmacia Biotech UK Ltd, Little Chalfont, UK).

2.5. Statistical Analysis. Statistical comparisons between $\mathrm{Hp} 1-1: \mathrm{Hb}$ and $\mathrm{Hp} 2-2: \mathrm{Hb}$ groups were performed using an unpaired Student's $t$-test. Multiple group comparisons of pSer473Akt activity were performed using a one way ANOVA with a Student-Newman-Keuls posttest. Statistical analysis was performed using GraphPad Prism (GraphPad Prism Software, Inc., San Diego, CA), and significance was assumed at $P<0.05$.

\section{Results}

3.1. Impaired IL-10 Secretion following CD163-Dependent Scavenging of Hp2-2: Hb. IL-10 induction has been linked to CD163 receptor engagement $[8,13,14,17,18]$. Differential IL10 responses by $\mathrm{CD}_{163}{ }^{+}$macrophages were therefore examined in the presence of polymorphic $\mathrm{Hp}: \mathrm{Hb}$ complexes. The addition of $\mathrm{Hp}: \mathrm{Hb}$ complexes across a range of concentrations to in vitro differentiated $\mathrm{CD}_{163}{ }^{+}$macrophages revealed significantly impaired IL-10 induction in the case of Hp2-2. At concentrations within the range of plasma haptoglobin $(0.1-2 \mathrm{~g} / \mathrm{L})[18,25]$, the IL-10 response to $\mathrm{Hp} 2-2: \mathrm{Hb}$ was significantly diminished compared to Hp1-1:Hb (e.g., at $1 \mathrm{~g} / \mathrm{L}$ : $3509 \pm 169 \mathrm{pg} / \mathrm{mL}$ versus $6739 \pm 678, P<0.01$; Figure 1(a)). The timecourse of IL-10 induction to either type of $\mathrm{Hp}: \mathrm{Hb}$ complex was relatively slow, with a peak at $48 \mathrm{~h}$ (Figure $1(\mathrm{~b})$ ). Consistent with the slow kinetic, IL-10 secretion required prior transcription and protein synthesis, as it was abrogated in the presence of actinomycin D or cycloheximide (data not shown). In agreement with previous observations $[8,15,17]$ and again repeated here, IL-10 induction required formation of a $\mathrm{Hp}: \mathrm{Hb}$ protein complex, since neither $\mathrm{Hb}$ alone, nor $\mathrm{Hp}$ alone yielded significant IL-10 secretion (Figure 1(c)). A panel 


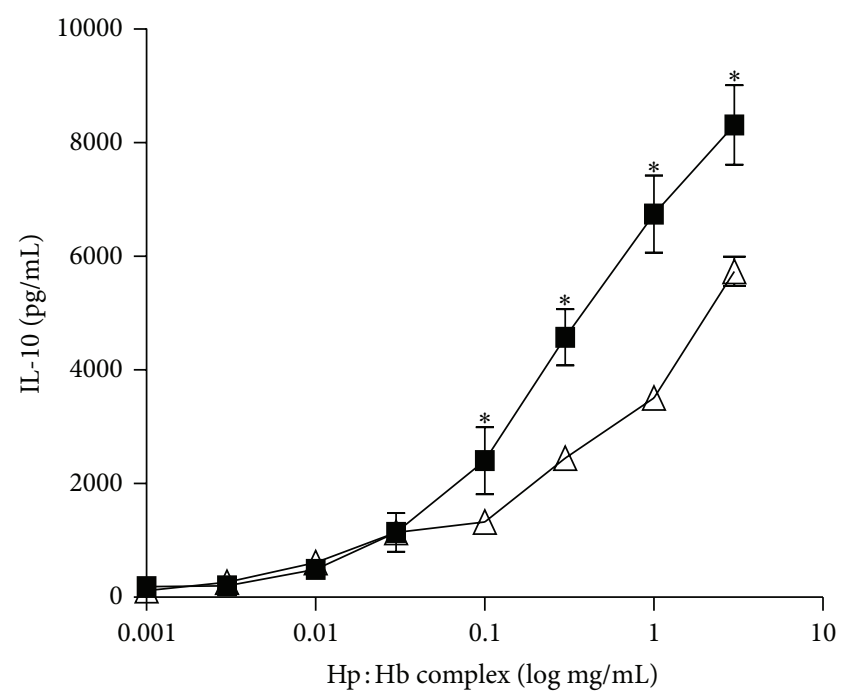

Hp 1-1

$\triangle$ Hp 2-2

(a)

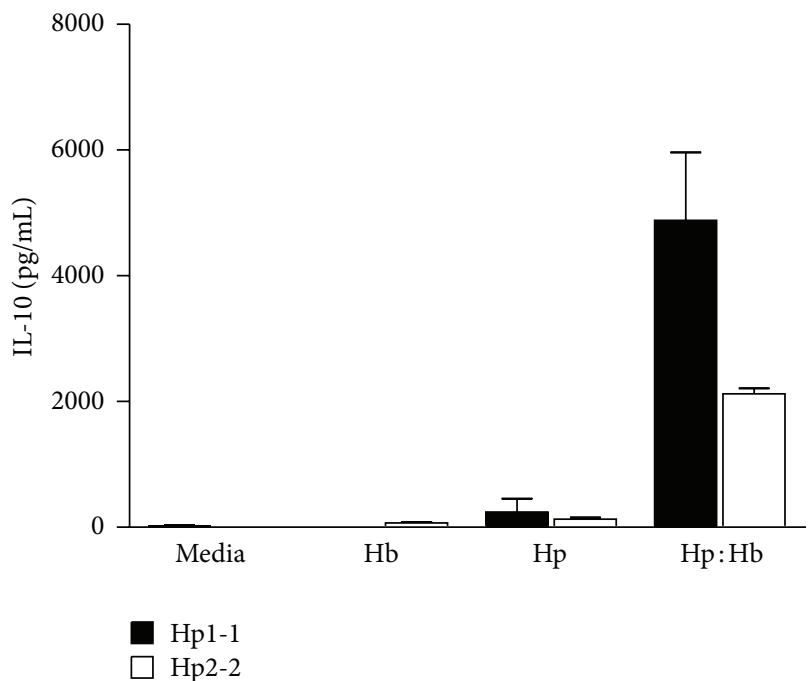

(c)

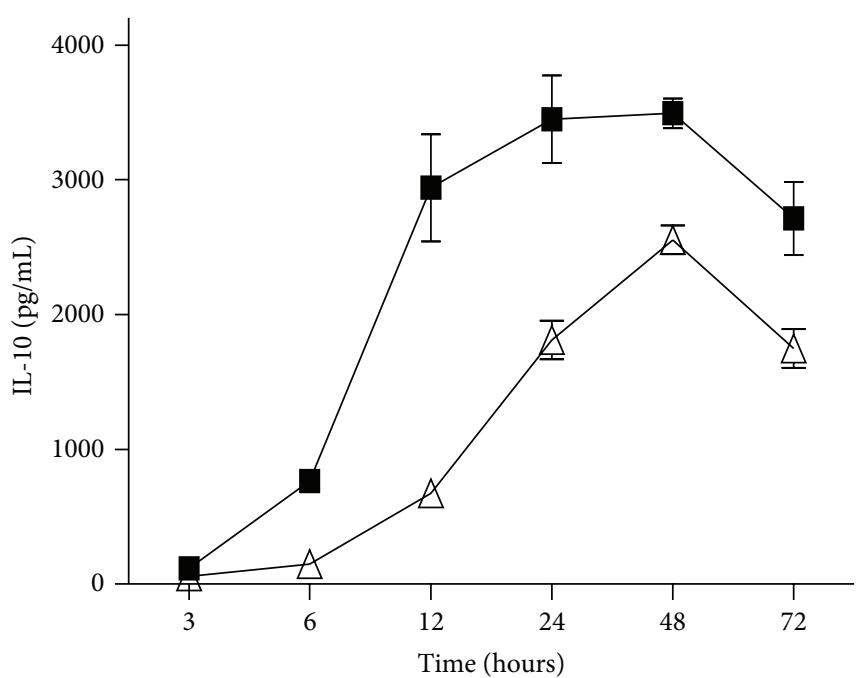

Hp 1-1

$\triangle$ Hp 2-2
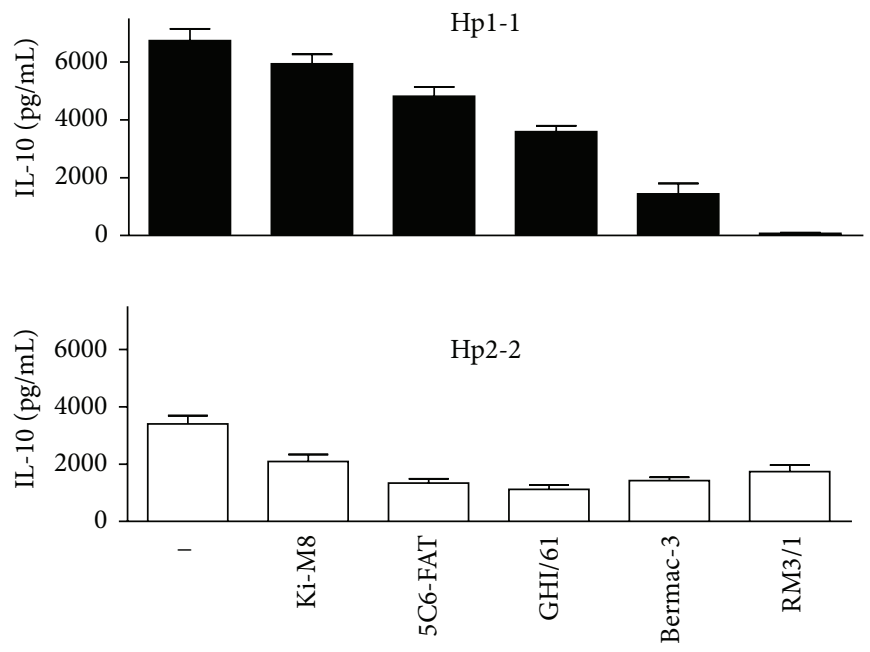

(d)

FIGURE 1: CD163-dependent IL-10 secretion following scavenging of polymorphic Hp:Hb complexes. (a) Effect of increasing concentrations of polymorphic $\mathrm{Hp}: \mathrm{Hb}$ complexes on IL-10 secretion by $\mathrm{CD}_{163^{+}}$human monocyte-derived macrophages at 24 h. (b) Timecourse of IL-10 secretion following addition of polymorphic $\mathrm{Hp}: \mathrm{Hb}$ complexes at $1 \mathrm{mg} / \mathrm{mL}$. (c) IL-10 secretion by CD $163^{+}$macrophages stimulated with $\mathrm{Hb}$ alone, Hp1-1, Hp2-2, or polymorphic Hp:Hb complexes $(1 \mathrm{mg} / \mathrm{mL}$ ) at $24 \mathrm{~h}$. (d) Effect of a panel of anti-CD163 antibodies (20 $\mu \mathrm{g} / \mathrm{mL}) \mathrm{on} \mathrm{IL-10}$ secretion induced by polymorphic $\mathrm{Hb}: \mathrm{Hp}$ complexes $(1 \mathrm{mg} / \mathrm{mL})$ at $24 \mathrm{~h}$. All IL-10 assays were carried out in duplicate, and results are expressed as mean IL-10 $(\mathrm{pg} / \mathrm{mL}) \pm$ SEM from $n=3-9$ experiments. ${ }^{*} P<0.01$.

of five anti-CD163 antibodies showed a similar inhibitory profile to either type of complex with the notable exception of the function blocking anti-CD163 antibody RM3/1 [8]. RM3/1 abolished IL-10 secretion in response to Hp1-1:Hb but only weakly inhibited the response to Hp2-2:Hb (Figure 1(d)).

3.2. Signaling Pathways Linked to CD163-Dependent $\mathrm{Hp}: \mathrm{Hb}$ Scavenging. The IL-10 response to either type of Hp required phagocytosis of complexes, since it was blocked in the presence of the phagocytosis inhibitor colchicine (Figure 2(a)). A screen of PI-3K, PKC, p42/44 MAP kinase, and p38 MAP kinase signaling pathway inhibitors revealed that the IL-10 response to $\mathrm{Hp}: \mathrm{Hb}$ complex scavenging was dose dependently inhibited by the PI-3K inhibitor LY294002 (Figure 2(b)). This was true for $\mathrm{Hp} 2-2: \mathrm{Hb}$ as well as $\mathrm{Hp1}-1: \mathrm{Hb}$ complexes. The pan-PKC inhibitor bisindolylmaleimide, the p42/44 MAP kinase inhibitor U0126, and p38 MAP kinase 

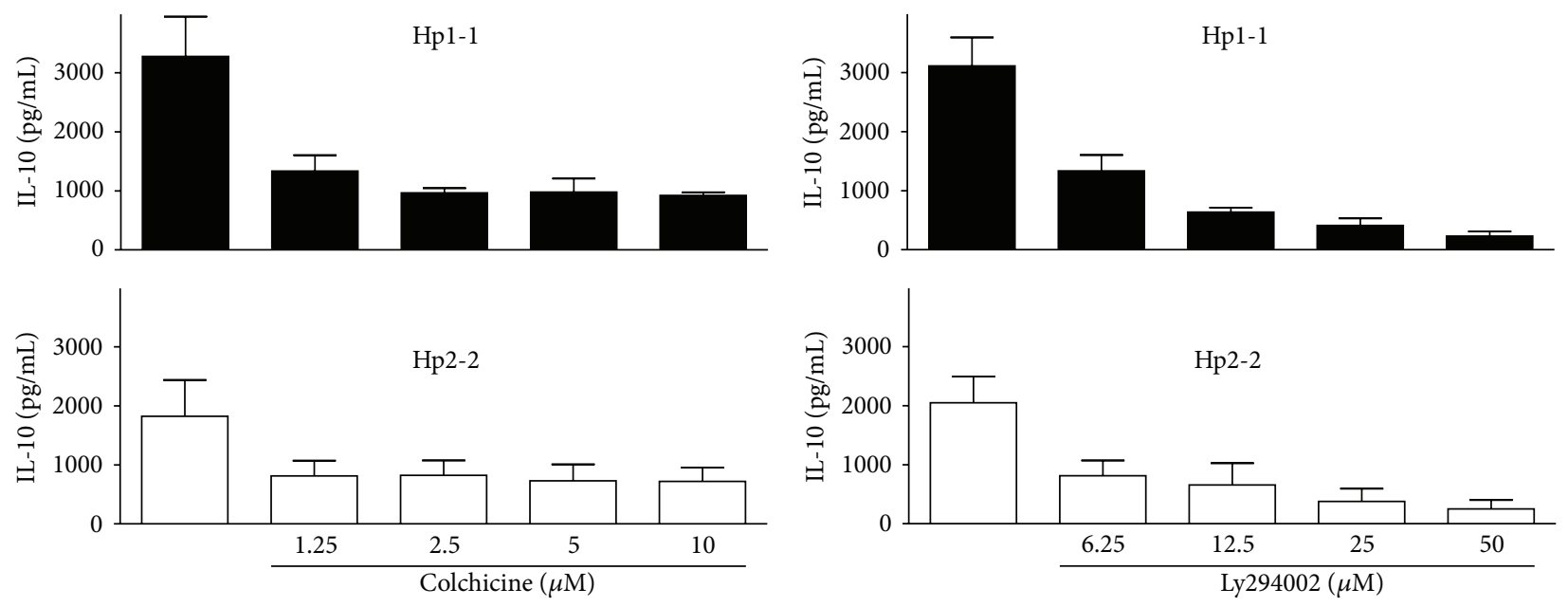

(a)

(b)

FIGURE 2: Effect of pharmacological inhibitors on Hp dependent IL-10 secretion. Effect of (a) colchicine (1.25-10 $\mu \mathrm{mol} / \mathrm{L})$ and (b) Ly294002 $(6.25-50 \mu \mathrm{mol} / \mathrm{L})$ on IL-10 release by $\mathrm{CD} 163^{+}$human monocyte-derived macrophages stimulated with polymorphic Hp:Hb complexes $(1 \mathrm{mg} / \mathrm{mL})$ for $24 \mathrm{~h}$. Results are expressed as mean IL-10 (pg/mL) \pm SEM from $n=3-4$ experiments.

inhibitor SB203580, used at a concentration range known to inhibit cytokine secretion in the same cell preparation against calcific microcrystals [24], had no effect on IL-10 secretion (data not shown).

To further explore signaling pathways linked to CD163 downstream of PI-3 kinase, phosphorylation of Akt was investigated. Phosphorylation of Akt at serine 473 (pSer473Akt) was examined by Western blot as well as phosphoSer473Akt-specific ELISA: both assays showed phosphorylation of Ser473Akt and this was inhibited significantly by anti-CD163 antibody RM3/1 or colchicine (Figures 3(a) and 3(b)). Akt phosphorylation required upstream PI-3Kinase activation, as it was abrogated in the presence of PI-3Kinase inhibitor Ly294002 (Figures 3(a) and 3(b)). Akt phosphorylation was markedly impaired following scavenging of $\mathrm{Hp} 2-2: \mathrm{Hb}$ complexes compared to $\mathrm{Hp} 1-1: \mathrm{Hb}$, across a broad concentration range (e.g., at $1 \mathrm{~g} / \mathrm{L}$ : $0.20 \pm$ $0.02 \mathrm{U} / \mathrm{mg}$ versus $0.50 \pm 0.10, P<0.05$; Figure $3(\mathrm{c})$ ).

\section{Discussion}

The central role of $\mathrm{Hp}$ in complexing free $\mathrm{Hb}$ and mediating its clearance through CD163 led us to consider whether the Hp2-2 genotype, clinically associated with cardiovascular complications, may show impaired anti-inflammatory signaling engagement downstream of CD163. The model chosen for this work (human monocytes differentiated for 7 days in culture) benefits from the absence of exogenously added differentiating agents (e.g., steroids) and has been validated against human macrophages differentiated in vivo: equivalent functional responses to $\mathrm{Hp}: \mathrm{Hb}$ scavenging were noted in $\mathrm{CD}_{163}{ }^{+}$macrophages recovered from human skin blisters during the cutaneous inflammatory response to cantharidin [8]. The CD163/Akt/IL-10 axis identified here adds to a growing understanding that homeostatic signalling pathways may be triggered in $\mathrm{CD}_{163}{ }^{+}$macrophages during hemoglobin scavenging and that these are impaired in the case of polymorphic Hp2-2 protein. Our data add a cellular dimension to the innate antioxidant properties recognised for $\mathrm{Hp}$ variants (Hp1-1 superior to $\mathrm{Hp} 2-1$ superior to $\mathrm{Hp} 2-2$ ) $[4,26,27]$ and suggest a plausible new disease mechanism linking Hp2-2 with atherothrombosis.

Our findings are consistent with previous reports on the relative inability of $\mathrm{Hp} 2-2: \mathrm{Hb}$ complexes to trigger IL10 responses $[17,18]$ and may explain why previous reports noted an apparent lack of CD163 receptor usage by polymorphic Hp2-2:Hb variants $[17,18]$. In those studies, RM3/1 was employed as the blocking antibody. Here we confirm that RM3/1 is the most potent blocking antibody against Hp1$1: H b$ induced IL-10 secretion, out of a screen of five antiCD163 antibodies (RM3/1, Ki-M8, 5C6-FAT, GHI/61, and Bermac-3). However, RM3/1 exhibited only weak blocking against $\mathrm{Hp} 2-2: \mathrm{Hb}$ complexes. Hence, it is not likely that $\mathrm{Hp} 2-2: \mathrm{Hb}$ signals through a different (unknown) receptor pathway. The evidence presented here with other blocking antibodies (Bermac3 and GHI/61) as well as the phagocytosis inhibitor colchicine strongly suggests that both types of $\mathrm{Hp}: \mathrm{Hb}$ complexes are endocytosed via CD163.

Results from the panel of blocking antibodies also have implications for the way in which signals are generated through CD163. The elegant structure-function analysis of CD163 protein [28], which combined ligand binding studies in solution with a comprehensive epitope map of ten antiCD163 antibodies, failed to detect any direct ligand-blocking property for RM3/1. Only Ki-m8 and Edhu-1, mapping to the ligand binding scavenger receptor cysteine-rich (SRCR) domain 3, directly inhibited Hp:Hb binding to CD163 in solution. Since these same two antibodies behaved as agonistic antibodies when added to cells $[8,13,14]$, this supports the concept that cross-linking of two or more ligand-binding 


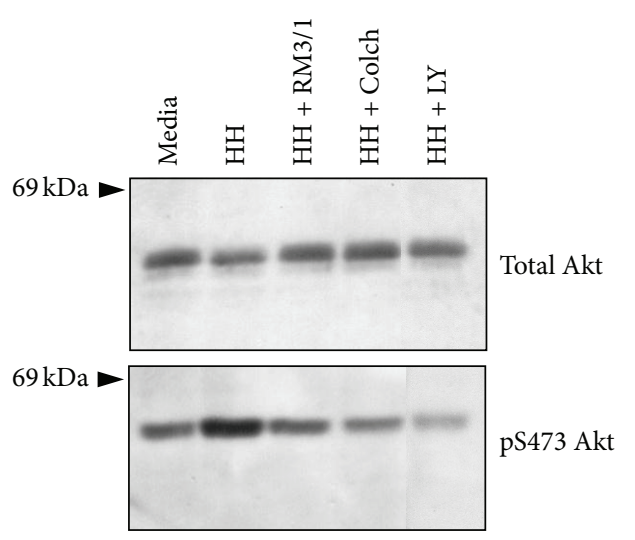

(a)

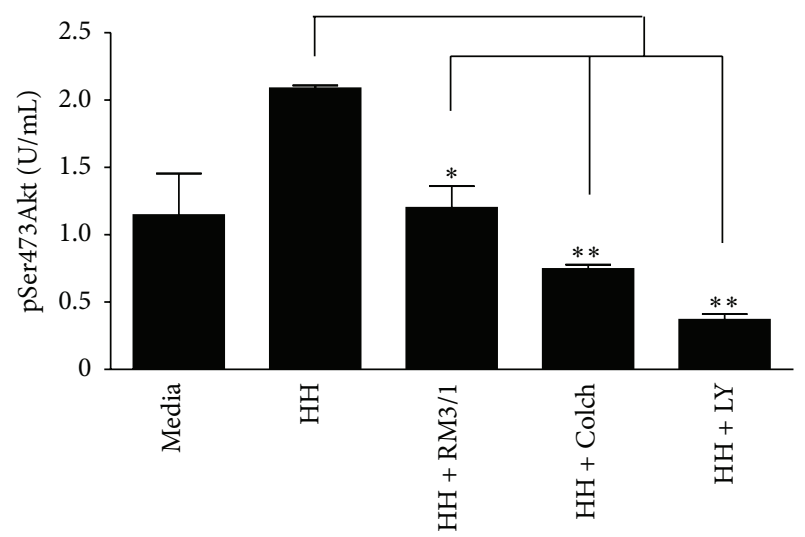

(b)

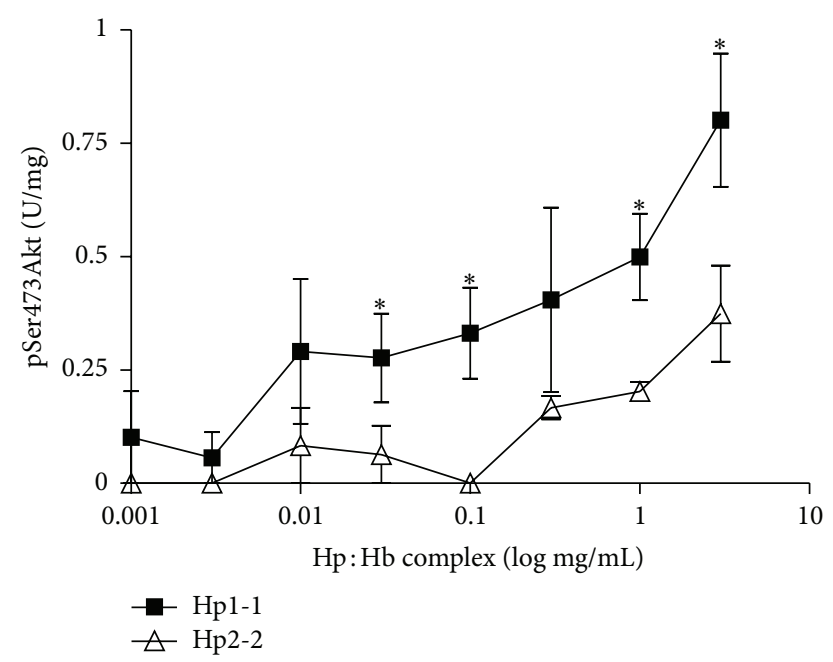

(c)

FiguRE 3: Hp:Hb scavenging-dependent Akt phosphorylation. (a) Representative Western blot showing phosphorylated Akt (pSer473) and total Akt in lysates from macrophages stimulated with $\mathrm{Hpl}-1: \mathrm{Hb}(1 \mathrm{mg} / \mathrm{mL} ; \mathrm{HH}$ in legend) for 20 minutes in the presence and absence of blocking anti-CD163 antibody clone RM3/1 $(20 \mu \mathrm{g} / \mathrm{mL}$; RM3/1 in legend), colchicine $(2.5 \mu \mathrm{mol} / \mathrm{L}$; colch in legend), or phosphoinositol 3kinase inhibitor Ly294002 (12.5 $\mu \mathrm{mol} / \mathrm{L}$; Ly in legend). (b) Quantitative ELISA determination of Akt (pSer473) phosphorylation in cell lysates from macrophages treated as in (a) above. (c) Comparison of Hp2-2:Hb versus Hp1-1:Hb scavenging on Akt phosphorylation in cell lysates, as measured by pSer473Akt-specific ELISA. Results in (b) and (c) are expressed as pSer473Akt (U/mg) \pm SEM from three experiments. ${ }^{*} P<0.05,{ }^{* *} P<0.01$.

domains on CD163 must be required for signal generation, as originally proposed [15]. Out of the panel of ten anti-CD163 antibodies previously mapped, RM3/1 uniquely mapped to the last SRCR domain (domain 9, immediately proximal to the plasma membrane) [28]. This suggests that its function blocking property relates to an ability to block dimerization of CD163 at the membrane following cross-linking with native Hp1-1:Hb holo-dimer. Since Hp2-2 exists as a larger multimer, consisting of 3-8 subunits, this might explain why RM3/1 only partially blocks IL-10 signal production in response to the multimeric complex.

We describe a hitherto unappreciated signalling pathway following $\mathrm{Hp}: \mathrm{Hb}$ complex endocytosis, requiring Ser473Akt phosphorylation and culminating in IL-10 secretion. We recognise the limitations posed through the use of a pharmacologic inhibitor of PI-3 kinase, but phosphorylation of Ser473Akt was verified both at Western blot and quantitative intracellular ELLISA levels. Adenoviral infection approaches to further dissect the signalling pathway were not successful, since infection even with empty vectors triggered phosphorylation of Akt, therefore ruling out this approach (data not shown). Past studies that used adenovirus to investigate signalling pathways in macrophages used differentiating agents not compatible with the $\mathrm{CD}_{163}{ }^{+}$endpoint and our cells may therefore have behaved differently [29].

Studies carried out prior to the identification of $\mathrm{Hp}: \mathrm{Hb}$ as ligand for CD163 employed a cross-linking anti-CD163 antibody, EDhu-1, and reported IL-6 secretion that was casein kinase II and PKC dependent $[13,14]$. Secretion of IL-6 in our hands, in response to $\mathrm{Hp}: \mathrm{Hb}$, was not specific to $\mathrm{CD} 163$, since it did not require $\mathrm{Hp}$ complex formation with $\mathrm{Hb}$, did not require an intact microtubular assembly for phagocytosis, and was not blocked by function-blocking antibodies or a PI-3K inhibitor (data not shown). Our own 
results do not therefore support IL-6 as an effector molecule downstream of CD163, whereas IL-10 was confirmed and was Hp genotype dependent. Alternative receptor mechanisms and endotoxin contamination were excluded as confounding sources of intracellular signals. Mac-1 was eliminated as a possible alternative candidate receptor for haptoglobin [30]. Endotoxin levels in all cell cultures were below $5 \mathrm{pg} / \mathrm{mL}$ and inhibition of IL-10 secretion by phagocytic inhibitors also ruled out endotoxin as a possible trigger. Our previous work has shown that above a threshold concentration of $>10 \mathrm{ng} / \mathrm{mL}$ of $\mathrm{Hp}: \mathrm{Hb}$ complexes, all or none commitment to the $\mathrm{CD} 163^{+}$ phenotype occurs via an IL-10/CD163 feedback loop [31]. This scenario is consistent with observations that $\mathrm{CD} 163^{+}$ macrophages closely associate with areas of hemorrhage [10, 31].

In summary, the present study has identified an PI3/Akt signaling pathway contributing to IL-10 secretion via $\mathrm{CD} 63^{+}$-dependent hemoglobin scavenging, a pathway that is impaired during the scavenging of polymorphic Hp22 complexes. This adds not only to our understanding of cardiovascular disease susceptibility for Hp2-2 but may also be relevant to the protection of the vasculature and kidneys from heme-mediated oxidative injury secondary to hemolysis [32-35], especially in predisposing conditions like diabetes, sickle cell anemia, or leukaemia treatment $[16,36,37]$. In conjunction with previous observations in atherosclerotic plaques $[5,12,31]$, we propose that $\mathrm{CD} 163^{+}$ macrophages may act as a homeostatic brake on plaque inflammation secondary to hemorrhage, in an Hp genotype dependent manner.

\section{Conflict of Interests}

The authors declare no conflict of interests.

\section{Acknowledgments}

This work was supported by the British Heart Foundation and the Barbados Diabetes Foundation.

\section{References}

[1] P. R. Moreno and V. Fuster, "New aspects in the pathogenesis of diabetic atherothrombosis," Journal of the American College of Cardiology, vol. 44, no. 12, pp. 2293-2300, 2004.

[2] F. D. Kolodgie, H. K. Gold, A. P. Burke et al., "Intraplaque hemorrhage and progression of coronary atheroma," The New England Journal of Medicine, vol. 349, no. 24, pp. 2316-2325, 2003.

[3] N. Takaya, C. Yuan, B. Chu et al., "Presence of intraplaque hemorrhage stimulates progression of carotid atherosclerotic plaques: a high-resolution magnetic resonance imaging study," Circulation, vol. 111, no. 21, pp. 2768-2775, 2005.

[4] A. P. Levy, J. E. Levy, S. Kalet-Litman et al., "Haptoglobin genotype is a determinant of iron, lipid peroxidation, and macrophage accumulation in the atherosclerotic plaque," Arteriosclerosis, Thrombosis, and Vascular Biology, vol. 27, no. 1, pp. 134-140, 2007.
[5] A. V. Finn, M. Nakano, R. Polavarapu et al., "Hemoglobin directs macrophage differentiation and prevents foam cell formation in human atherosclerotic plaques," Journal of the American College of Cardiology, vol. 59, no. 2, pp. 166-177, 2012.

[6] J. J. Boyle, "Heme and haemoglobin direct macrophage Mhem phenotype and counter foam cell formation in areas of intraplaque haemorrhage," Current Opinion in Lipidology, vol. 23, no. 5, pp. 453-461, 2012.

[7] J. J. Boyle, H. A. Harrington, E. Piper et al., "Coronary intraplaque hemorrhage evokes a novel atheroprotective macrophage phenotype," American Journal of Pathology, vol. 174, no. 3, pp. 1097-1108, 2009.

[8] P. Philippidis, J. C. Mason, B. J. Evans et al., "Hemoglobin scavenger receptor CD163 mediates interleukin-10 release and heme oxygenase-1 synthesis: antiinflammatory monocytemacrophage responses in vitro, in resolving skin blisters in vivo, and after cardiopulmonary bypass surgery," Circulation Research, vol. 94, no. 1, pp. 119-126, 2004.

[9] N. G. Abraham and G. Drummond, "CD163-mediated hemoglobin-heme uptake activates macrophage HO-1, providing an antiinflammatory function," Circulation Research, vol. 99, no. 9, pp. 911-914, 2006.

[10] C. A. Schaer, G. Schoedon, A. Imhof, M. O. Kurrer, and D. J. Schaer, "Constitutive endocytosis of CD163 mediates hemoglobin-heme uptake and determines the noninflammatory and protective transcriptional response of macrophages to hemoglobin," Circulation Research, vol. 99, no. 9, pp. 943-950, 2006.

[11] J. J. Boyle, M. Johns, T. Kampfer et al., "Activating transcription factor 1 directs Mhem atheroprotective macrophages through coordinated iron handling and foam cell protection," Circulation Research, vol. 110, no. 1, pp. 20-33, 2012.

[12] J. J. Boyle, M. Johns, J. Lo et al., "Heme induces heme oxygenase 1 via Nrf2: role in the homeostatic macrophage response to intraplaque hemorrhage," Arteriosclerosis, Thrombosis, and Vascular Biology, vol. 31, no. 11, pp. 2685-2691, 2011.

[13] M. M. Van Den Heuvel, C. P. Tensen, J. H. van As et al., "Regulation of CD163 on human macrophages: cross-linking of CD163 induces signaling and activation," Journal of Leukocyte Biology, vol. 66, no. 5, pp. 858-866, 1999.

[14] M. Ritter, C. Buechler, M. Kapinsky, and G. Schmitz, "Interaction of CD163 with the regulatory subunit of casein kinase II, (CKII) and dependence of CD163 signaling on CKII and protein kinase C," European Journal of Immunology, vol. 31, no. 4, pp. 999-1009, 2001.

[15] M. Kristiansen, J. H. Graversen, C. Jacobsen et al., "Identification of the haemoglobin scavenger receptor," Nature, vol. 409, no. 6817, pp. 198-201, 2001.

[16] R. Asleh, S. Marsh, M. Shilkrut et al., "Genetically determined heterogeneity in hemoglobin scavenging and susceptibility to diabetic cardiovascular disease," Circulation Research, vol. 92, no. 11, pp. 1193-1200, 2003.

[17] J. Guetta, M. Strauss, N. S. Levy, L. Fahoum, and A. P. Levy, "Haptoglobin genotype modulates the balance of Th1/Th2 cytokines produced by macrophages exposed to free hemoglobin," Atherosclerosis, vol. 191, no. 1, pp. 48-53, 2007.

[18] M. Strauss and A. P. Levy, "Regulation of CD163 associated casein kinase II activity is haptoglobin genotype dependent," Molecular and Cellular Biochemistry, vol. 317, no. 1-2, pp. 131$135,2008$.

[19] A. P. Levy, I. Hochberg, K. Jablonski et al., "Haptoglobin phenotype is an independent risk factor for cardiovascular 
disease in individuals with diabetes: the strong heart study," Journal of the American College of Cardiology, vol. 40, no. 11, pp. 1984-1990, 2002.

[20] M. Suleiman, D. Aronson, R. Asleh et al., "Haptoglobin polymorphism predicts 30-day mortality and heart failure in patients with diabetes and acute myocardial infarction," Diabetes, vol. 54, no. 9, pp. 2802-2806, 2005.

[21] U. Milman, S. Blum, C. Shapira et al., "Vitamin E supplementation reduces cardiovascular events in a subgroup of middleaged individuals with both type 2 diabetes mellitus and the haptoglobin 2-2 genotype: a prospective double-blinded clinical trial," Arteriosclerosis, Thrombosis, and Vascular Biology, vol. 28, no. 2, pp. 341-347, 2008.

[22] L. E. Cahill, A. P. Levy, S. E. Chiuve et al., "Haptoglobin genotype is a consistent marker of coronary heart disease risk among individuals with elevated glycosylated hemoglobin," Journal of the American College of Cardiology, vol. 61, no. 7, pp. 728-737, 2013.

[23] S. Kalet-Litman, P. R. Moreno, and A. P. Levy, "The haptoglobin 2-2 genotype is associated with increased redox active hemoglobin derived iron in the atherosclerotic plaque," Atherosclerosis, vol. 209, no. 1, pp. 28-31, 2010.

[24] I. Nadra, J. C. Mason, P. Philippidis et al., "Proinflammatory activation of macrophages by basic calcium phosphate crystals via protein kinase $\mathrm{C}$ and MAP kinase pathways: a vicious cycle of inflammation and arterial calcification?" Circulation Research, vol. 96, no. 12, pp. 1248-1256, 2005.

[25] I. Kasvosve, Z. A. R. Gomo, I. T. Gangaidzo et al., "Reference range of serum haptoglobin is haptoglobin phenotypedependent in blacks," Clinica Chimica Acta, vol. 296, no. 1-2, pp. 163-170, 2000.

[26] M. Melamed-Frank, O. Lache, B. I. Enav et al., "Structurefunction analysis of the antioxidant properties of haptoglobin," Blood, vol. 98, no. 13, pp. 3693-3698, 2001.

[27] V. V. Bamm, V. A. Tsemakhovich, M. Shaklai, and N. Shaklai, "Haptoglobin phenotypes differ in their ability to inhibit heme transfer from hemoglobin to LDL," Biochemistry, vol. 43, no. 13, pp. 3899-3906, 2004.

[28] M. Madsen, H. J. Møller, M. J. Nielsen et al., "Molecular characterization of the haptoglobin-hemoglobin receptor CD163: ligand binding properties of the scavenger receptor cysteinerich domain region," The Journal of Biological Chemistry, vol. 279, no. 49, pp. 51561-51567, 2004.

[29] J. Bondeson, K. A. Browne, F. M. Brennan, B. M. J. Foxwell, and M. Feldmann, "Selective regulation of cytokine induction by adenoviral gene transfer, of I $\kappa \mathrm{B} \alpha$ into human macrophages: lipopolysaccharide-induced, but not zymosan-induced, proinflammatory cytokines are inhibited, but IL-10 is nuclear factor$\kappa \mathrm{B}$ independent," Journal of Immunology, vol. 162, no. 5, pp. 2939-2945, 1999.

[30] S. M. El Ghmati, E. M. Van Hoeyveld, J. A. G. Van Strijp, J. L. Ceuppens, and E. A. M. Stevens, "Identification of haptoglobin as an alternative ligand for CD11b/CD18," Journal of Immunology, vol. 156, no. 7, pp. 2542-2552, 1996.

[31] J. J. Boyle, H. A. Harrington, E. Piper et al., "Coronary intraplaque hemorrhage evokes a novel atheroprotective macrophage phenotype," American Journal of Pathology, vol. 174, no. 3, pp. 1097-1108, 2009.

[32] R. Asleh and A. P. Levy, "In vivo and in vitro studies establishing haptoglobin as a major susceptibility gene for diabetic vascular disease," Vascular Health and Risk Management, vol. 1, no. 1, pp. 19-28, 2005.
[33] S. K. Lim, H. Kim, S. K. Lim et al., "Increased susceptibility in Hp knockout mice during acute hemolysis," Blood, vol. 92, no. 6, pp. 1870-1877, 1998.

[34] R. P. Rother, L. Bell, P. Hillmen, and M. T. Gladwin, "The clinical sequelae of intravascular hemolysis and extracellular plasma hemoglobin: a novel mechanism of human disease," Journal of the American Medical Association, vol. 293, no. 13, pp. 16531662, 2005.

[35] R. Asleh, F. M. Nakhoul, R. Miller-Lotan et al., "Poor lysosomal membrane integrity in proximal tubule cells of haptoglobin 2-2 genotype mice with diabetes mellitus," Free Radical Biology \& Medicine, vol. 53, no. 4, pp. 779-786, 2012.

[36] C. D. Reiter, X. Wang, J. E. Tanus-Santos et al., "Cell-free hemoglobin limits nitric oxide bioavailability in sickle-cell disease," Nature Medicine, vol. 8, no. 12, pp. 1383-1389, 2002.

[37] M. B. Maniecki, H. Hasle, L. Friis-Hansen et al., "Impaired CD163-mediated hemoglobin-scavenging and severe toxic symptoms in patients treated with gemtuzumab ozogamicin," Blood, vol. 112, no. 4, pp. 1510-1514, 2008. 


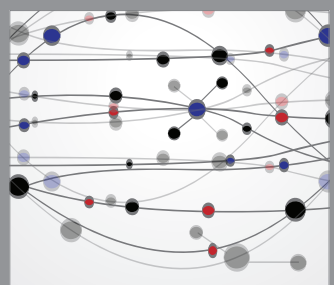

The Scientific World Journal
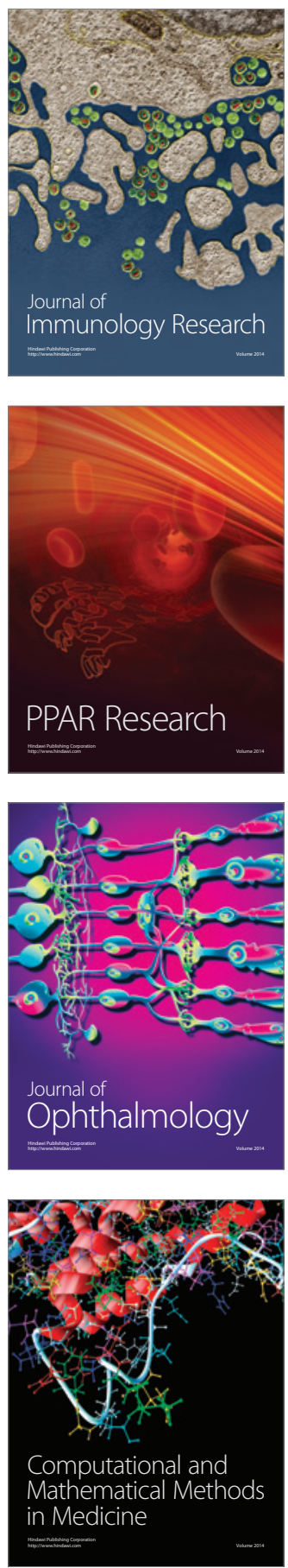

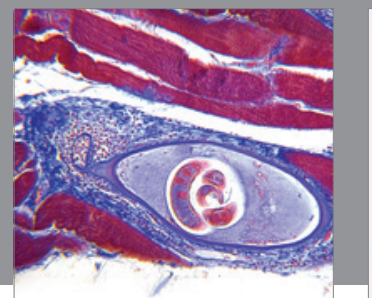

Gastroenterology

Research and Practice
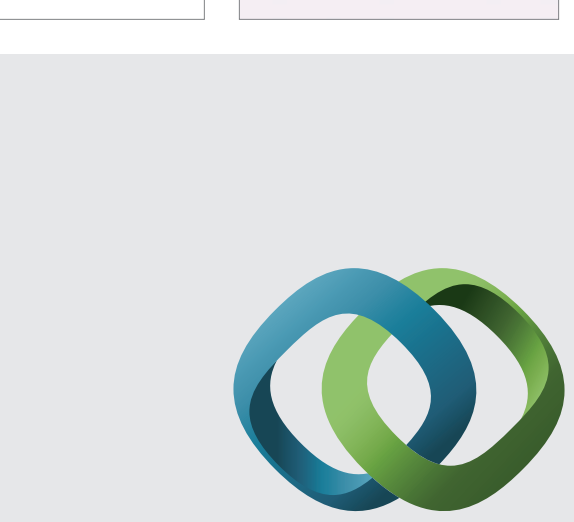

\section{Hindawi}

Submit your manuscripts at

http://www.hindawi.com
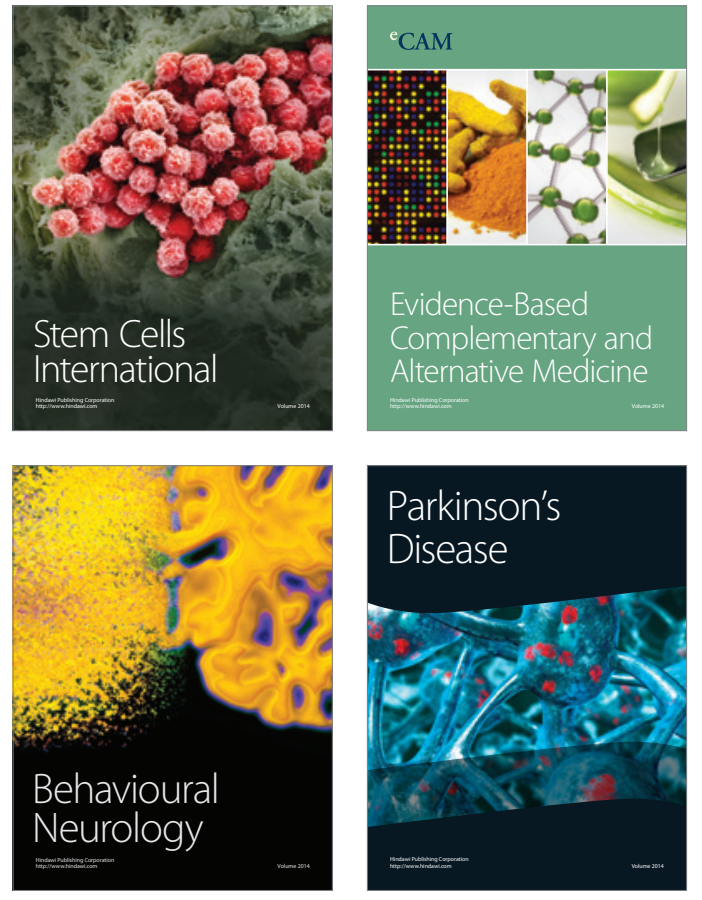
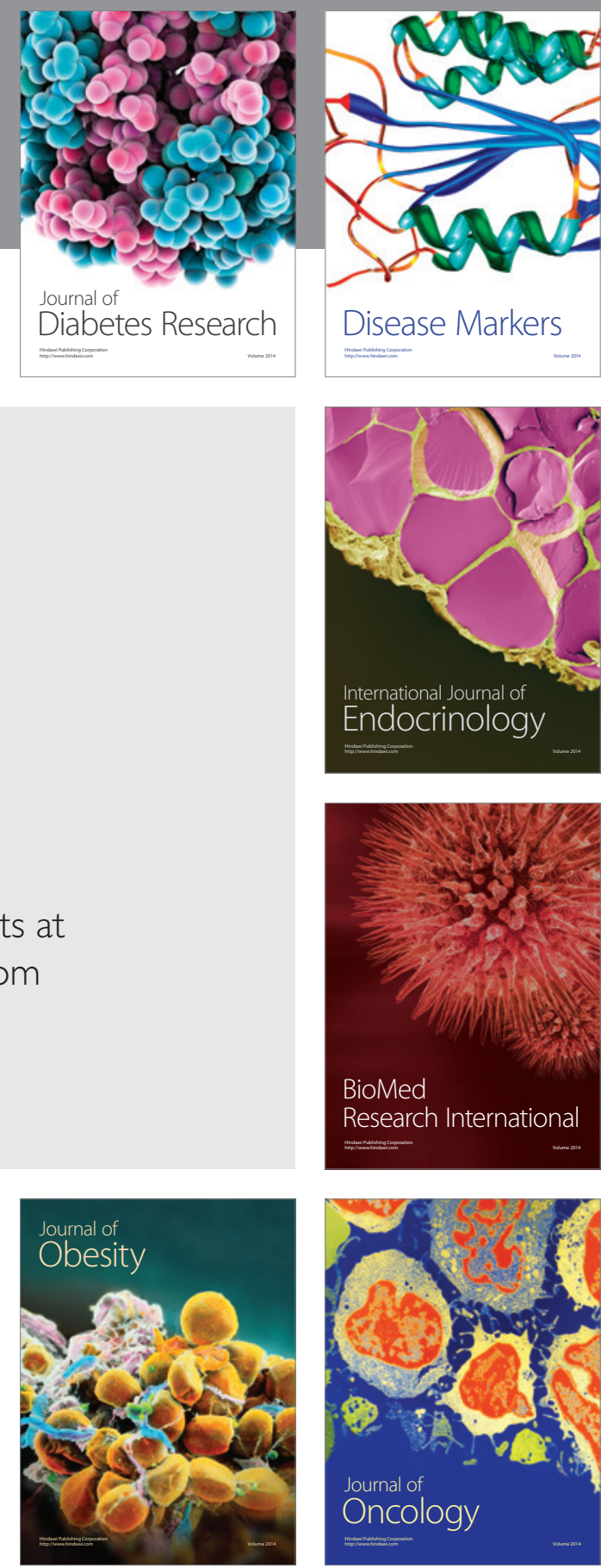

Disease Markers
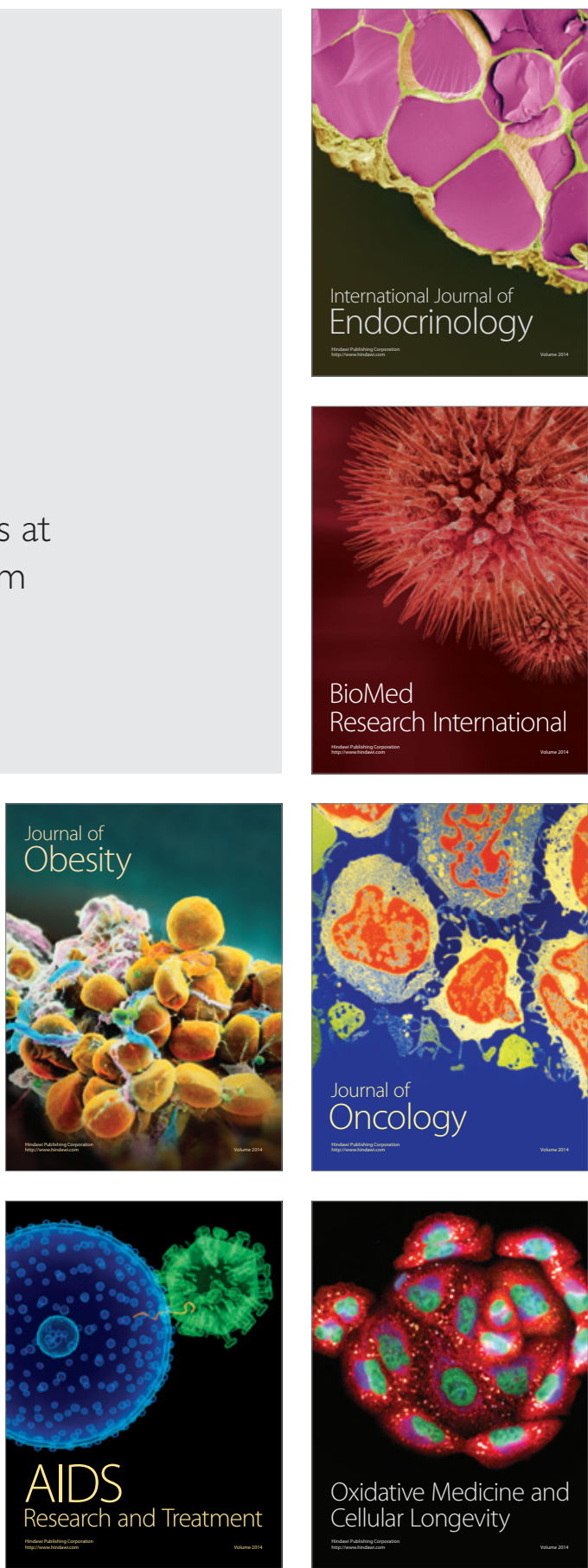